Rev. Bras. Saúde Prod. Anim., Salvador, v.16, n.3, p.699-711 jul./set.., $2015 \quad \underline{\text { http://www.rbspa.ufba.br }}$ ISSN 15199940

http://dx.doi.org/10.1590/S1519-99402015000300020

\title{
Eficiência bioeconômica de bovinos de corte em confinamento
}

\author{
Bioeconomic efficiency of beef cattle at feedlot
}

\author{
NICHELE, Evelyn Mangilli ${ }^{1}$; MORAES, Eduardo Henrique Bevitori Kling de ${ }^{2 *}$; \\ ARAÚJO, Cláudio Vieira de de $^{3}$ PINA, Douglas dos Santos²; MORAES, Kamila \\ Andreatta Kling de ${ }^{3}$, HOFFMANN, Alvair ${ }^{4}$
}

\author{
${ }^{1}$ Universidade Federal de Mato Grosso, Programa de Pós-Graduação em Ciência Animal, Cuiabá, Mato \\ Grosso, Brasil. \\ ${ }^{2}$ Universidade Federal de Mato Grosso, Instituto de Ciências Agrárias e Ambientais, Sinop, Mato Grosso, \\ Brasil. Pesquisador do INCT/Ciência Animal/CNPq \\ ${ }^{3}$ Universidade Federal de Mato Grosso, Instituto de Ciências Agrárias e Ambientais, Sinop, Mato \\ Grosso, Brasil. \\ ${ }^{4}$ Universidade Estadual Paulista, Programa de Pós-Graduação em Zootecnia, Jaboticabal, São Paulo, Brasil \\ *Endereço eletrônico para correspondência: edukling@ufmt.br
}

\section{RESUMO}

Objetivou-se avaliar o consumo de matéria seca, o ganho de peso total e a eficiência bioeconômica de bovinos de corte terminados em confinamento com diferentes pesos corporais ao início do confinamento nos períodos de seca e de chuvas. Registros de CMS, ganho de peso total (GPT), conversão alimentar (CA), eficiência alimentar (EA), tamanho de lote e tempo de permanência (TP) no confinamento de 13.686 bovinos de corte machos com predominância de sangue nelore terminados no ano de 2009, foram utilizados para discriminar possíveis diferenças quanto ao peso de entrada (até $350 \mathrm{~kg}$ ou acima de $350 \mathrm{~kg}$ ) e da época do ano (seca ou águas). Observou-se ausência de efeitos da época do ano (chuvas $\mathrm{x}$ seca) para o CMS e o GPT dos animais independente da classe de peso que apresentaram diferenças, com os animais mais leves apresentando menores CMS e maiores GPT e, consequentemente, maior eficiência que os animais mais pesados ao início do confinamento. $\mathrm{O}$ valor de compra por cabeça interfere altamente sobre valor por cabeça na saída do confinamento que é dependente do consumo de matéria seca e do ganho de peso total para maximização da receita.

Palavras-chave: consumo, desempenho, eficiência alimentar, variável canônica

\section{SUMMARY}

It was aimed to evaluate the dry matter intake (DMI), total weight gain (TWG) and the bioeconomic efficiency of feedlot beef cattle with different body weights in dry and rainy season. Records of DMI, TWG, feed conversion (FC), feed efficiency (EA), lot size and feedlot length of stay (LS) of 13,686 beef cattle males with predominance of Nelore blood finished the year 2009, were used to discriminate possible differences in input weight (up to $350 \mathrm{~kg}$ or above $350 \mathrm{~kg}$ ) and the season (dry or water). There was no effect on the season (rainy $x$ dry) to DMI and TWG of independent animals, regardless of weight class that showed differences, with the lighter animals presented lower DMI and higher TWG and therefore higher efficiency than animals with higher higher starting weights of feedlot. The purchase price per head affects highly on value per head for the exit of feedlot which is dependent on dry matter intake and total weight gain for maximizing revenue.

Keywords: canonical variable, feed efficiency, intake, performance 
Rev. Bras. Saúde Prod. Anim., Salvador, v.16, n.3, p.699-711 jul./set.., $2015 \quad \underline{\text { http://www.rbspa.ufba.br }}$

\section{INTRODUÇÃO}

O sistema de terminação em confinamentos de bovinos para corte tem sido ao longo dos anos alternativa para reduzir, significativamente, $\mathrm{o}$ tempo necessário para $\mathrm{o}$ abate dos animais e consequentemente aumentar a produtividade.

Neste contexto, a eficiência nutricional e econômica da terminação de bovinos em confinamento tem sido influenciada, entre outros fatores, pelo peso corporal dos animais na entrada do confinamento, pelo consumo de matéria seca (CMS) e pelo desempenho dos mesmos.

O desempenho animal sobre influência do CMS, que segundo Mertens (1994), varia conforme o animal, o alimento e as condições de alimentação e de clima. Em sistemas de terminação de bovinos em confinamento, verifica-se que a alimentação destaca-se como um dos itens mais onerosos, girando em torno de um terço do custo total (EZEQUIEL et al., 2006). Assim, o uso de animais mais eficientes na utilização dos nutrientes pode ter reflexos positivos quanto à redução dos custos de produção e consequentemente aumentar a lucratividade dos sistemas.

Neste sentido, o estudo da eficiência bioeconômica deve ser incluído nas avaliações de sistemas de confinamento devido sua importância quanto à determinação eficiência do ganho de peso dos animais.

Segundo Guidoni (1994), a eficiência bioeconômica é um índice de discriminação utilizado para revelar em que aspecto um grupo de animais é mais eficiente que o outro. Considerando que na pecuária de corte estes aspectos estão relacionados à eficiência de utilização do alimento ingerido para conversão em ganho de peso, a análise da eficiência bionutricional utiliza as variáveis ganho de peso e CMS de forma conjunta, em uma análise multivariada (PAZDIORA et al, 2013).

Assim, objetivou-se avaliar o consumo de matéria seca, o ganho de peso total e a eficiência bioeconômica de bovinos de corte terminados em confinamento com diferentes pesos de entrada nos períodos de seca e de chuvas.

\section{MATERIAL E MÉTODOS}

Registros de CMS, ganho de peso total (GPT), conversão alimentar (CA), eficiência alimentar (EA), tamanho de lote e tempo de permanência (TP) no confinamento de 13.686 bovinos de corte machos com predominância de sangue nelore terminados no ano de 2009, provenientes de um confinamento localizado na região Norte de Mato Grosso, foram utilizados para discriminar possíveis diferenças quanto ao peso de entrada e da época do ano (seca ou águas) em que foram confinados. Ressalta-se que o tamanho do lote não influenciou nas variáveis estudadas. Assim, pré ajustou-se os registros de GPT e CMS para o efeito PC inicial e TP no confinamento.

No tocante aos períodos, o período seco do ano foi considerado de 01 de abril a 30 de outubro, e o período chuvoso de 01 de novembro a 31 de março do respectivo ano. Quanto ao $\mathrm{PC}$ de entrada foram classificados em duas classes: animais leves, aqueles com peso próximo de $350 \mathrm{~kg}$ e animais pesados com peso acima de $350 \mathrm{~kg}$. Desta forma, foram compostos quatro tratamentos denominados como: período seco e animais leves - SL; período seco e animais pesados - SP; período chuvoso e animais leves - CL e período chuvoso e animais leves - $\mathrm{CP}$. 
Rev. Bras. Saúde Prod. Anim., Salvador, v.16, n.3, p.699-711 jul./set.., $2015 \quad \underline{\text { http://www.rbspa.ufba.br }}$ ISSN 15199940

Foi realizada análise de variância multivariada (MANOVA) para discriminação de diferenças entre tratamentos considerando-se conjuntamente o CMS e o GPT.

A abordagem da análise de variância multivariada foi utilizada para comparar vetores de médias, cujos dados normalmente são provenientes de delineamentos estatísticos (JOHNSON \& WICHERN, 1998). Um ponto relevante da análise multivariada é o aproveitamento da informação conjunta das variáveis envolvidas (REGAZZI, 2000).

O modelo estatístico utilizado foi o delineamento inteiramente casualizado (DIC) com $k$ tratamentos em que são medidas $p$ variáveis é: $Y_{i j}=\mu_{j}+t_{i}+e_{i j}$ $i=1,2, \ldots, k ; j=1,2, \ldots, p$ em que, $\mathrm{R}=$ indexador das variáveis; $Y_{i j}=$ valor observado da j-ésima variável sob o efeito do i-ésimo tratamento; $\mu_{j}=$ média geral da j-ésima variável; $t_{i j}=$ efeito do i-ésimo tratamento na j-ésima variável; $e_{i j}=$ efeito aleatório associado à observação $Y_{i j}$.

$\mathrm{Na}$ forma matricial o modelo estatístico foi: $Y=X \mathrm{~B}+\varepsilon$ em que, $Y=$ matriz de observações de dimensões $k \times p ; \quad X=$ matriz do delineamento de dimensões $k$ $\mathrm{x}(1+k) ; \mathrm{B}=$ matriz de parâmetros de dimensões $(1+k) \times p ; \varepsilon=$ matriz de erros de dimensões $k \times p$.

Para testar a hipótese de nulidade entre os vetores de médias de tratamentos, foram utilizados os critérios de Lambda de Wilks, Traço de Pillai, Traço de Hotelling-Lawley e Maior Raiz de Roy, adotando o nível de significância de 0,05 .

Posteriormente, com objetivo de compor um índice bionutricional, envolvendo o CMS e o GPT conjuntamente, adotou-se a análise discriminante por meio da utilização de variável canônica, estabelecendo a combinação linear dos pesos em novas variáveis (variáveis canônicas).

Realizou-se análise de correlação canônica de forma a associar as variáveis de produção (primeira variável canônica): custo de produção por arroba (CP@); valor de venda da arroba (VV@); custo da diária (CD), valor por cabeça na saída (VV/CAB) e lucro líquido do produto (LLíq) com as variáveis financeiras segunda variável canônica: valor de compra por cabeça (VC/CAB), lucro líquido por cabeça (LL/CAB) e valor por arroba de compra (VC@).

Foram calculadas, duas combinações lineares (variáveis canônicas), uma sobre cada conjunto de variáveis, de forma que os coeficientes de correlação de Pearson entre essas combinações fossem máximos. Em seguida, foram calculadas duas outras variáveis canônicas, uma sobre cada conjunto de variáveis, formando o segundo par canônico, com a condição de que as variáveis canônicas dentro de cada conjunto fossem ortogonais e assim sucessivamente (JOHNSON \& WICHERN, 1998).

Para verificar a significância da correlação canônica nos pares canônicos, foi utilizado o teste da razão de máxima verossimilhança, cuja estatística é a Lambda de Wilks, cujas expressões são apresentadas por Khattree \& Naik (2000).

\section{RESULTADOS E DISCUSSÃO}

Observou-se ausência de efeitos $(\mathrm{P}>0,05)$ da época do ano (chuvas $\mathrm{x}$ seca) para o CMS e o GPT dos animais independente da classe de peso que apresentaram diferenças, com os animais mais leves apresentando 
Rev. Bras. Saúde Prod. Anim., Salvador, v.16, n.3, p.699-711 jul./set.., $2015 \quad \underline{\text { http://www.rbspa.ufba.br }}$ ISSN 15199940

menores CMS e maiores GPT $(\mathrm{P}<0,05)$ que os animais mais pesados ao início do confinamento (Tabela 1).

É sabido que o PC é um determinante primário do consumo e, neste contexto, quanto maior o PC maior será a capacidade de ingestão de alimentos que também pode ser explicada pela maior demanda energética e protéica para mantença destes animais.

Segundo equações de predição de CMS recomendadas pelo BR-CORTE (VALADARES FILHO et al., 2010), as estimativas do CMS em função do ganho de peso mostrara uma curva com três segmentos distintos: fase de adaptação; platô; e declínio, as quais correspondem a adaptação ao confinamento ou ao ambiente, ao aumento do PC, e ao aumento do conteúdo de gordura corporal. De fato, a concentração de gordura na carcaça começa em ritmo lento no início do período de alimentação, mas acumula-se em um ritmo mais rápido em relação ao final do período de alimentação, (SIMPFENDORFER, 1974).

Neste contexto, embora o CMS em termos absolutos (kg/dia) aumente em função do PC, a capacidade de ingestão em relação ao $\mathrm{PC}(\mathrm{g} / \mathrm{kg} \mathrm{PC})$ decresce com o aumento do PC. (CHIZZOTTI \& CHIZZOTTI, 2014). Desta forma, segundo os autores, este fato indica que o aumento da capacidade ruminal não é diretamente proporcional ao acúmulo de PC.

Tabela 1. Consumo de matéria seca (CMS - kg/dia), ganho de peso total (GPT - kg), para as variáveis canônica (VC), conversão alimentar (CA) e eficiência alimentar (EA) para as combinações classes de peso e período do ano ${ }^{1}$

\begin{tabular}{lccccc}
\hline \multirow{2}{*}{ Item } & \multicolumn{2}{c}{ Período da seca } & \multicolumn{2}{c}{ Período das chuvas } & CV \\
\cline { 2 - 6 } & $<350 \mathrm{~kg}$ & $>350 \mathrm{~kg}$ & $<350 \mathrm{~kg}$ & $>350 \mathrm{~kg}$ & $(\%)$ \\
\hline CMS & $9,34^{\mathrm{a}}$ & $10,18^{\mathrm{b}}$ & $9,13^{\mathrm{a}}$ & $10,38^{\mathrm{b}}$ & 9,24 \\
GPT & $114,15^{\mathrm{a}}$ & $113,17^{\mathrm{b}}$ & $131,32^{\mathrm{a}}$ & $98,31^{\mathrm{b}}$ & 17,5 \\
VC1 & $3,75^{\mathrm{a}}$ & $6,39^{\mathrm{b}}$ & $3,27^{\mathrm{a}}$ & $5,99^{\mathrm{b}}$ & 5,09 \\
$\mathrm{CA}$ & $0,07^{\mathrm{a}}$ & $0,09^{\mathrm{b}}$ & $0,06^{\mathrm{a}}$ & $0,09^{\mathrm{b}}$ & 7,89 \\
EA & $14,64^{\mathrm{a}}$ & $10,81^{\mathrm{b}}$ & $15,55^{\mathrm{a}}$ & $11,18^{\mathrm{b}}$ & 6,29 \\
\hline
\end{tabular}

${ }^{\mathrm{C}}$ Médias seguidas de mesma letra na linha não diferem entre si pelo teste de Tukey ao nível de significância de 0,05 .

No tocante ao GPT, os menores valores observados nos animais mais pesados ao início do confinamento pode ser em parte explicado pela possível maior taxa de deposição de tecido adiposo que aumenta gradativamente após redução da taxa de deposição de tecido muscular. A síntese de tecido adiposo é mais lenta que a síntese de tecido muscular, pois é preciso apenas metade da energia para depositar a mesma quantidade de tecido muscular, se comparado ao tecido adiposo (MERSMANN, 1998).
Em estudo realizado com bovinos confinados, Cruz et al. (2004) observaram ganho de peso diário de 1,$56 ; 1,49$ e $1,44 \mathrm{~kg}$ animal ${ }^{-1} \mathrm{dia}^{-1}$, para animais com peso ao abate de 400, 440 e $480 \mathrm{Kg}$ de $\mathrm{PC}$, respectivamente.

Realizando-se a análise de variância multivariada (MANOVA) para o CMS e o GPT, verificou-se que os quatro critérios multivariados utilizados apontaram para diferenças significativas $(\mathrm{P}<0,05)$ entre vetores médias de tratamentos, sendo encontrados os valores de 0,$68 ; 0,33 ; 0,44$ e 0,39 , respectivamente, para os critérios 
Rev. Bras. Saúde Prod. Anim., Salvador, v.16, n.3, p.699-711 jul./set.., $2015 \quad \underline{\text { http://www.rbspa.ufba.br }}$

Lambda de Wilks, Traço de Pillai, Traço de Hotelling-Lawley e Maior Raiz de Roy. Assim, com base no resultado da MANOVA, procedeu-se o cálculo da primeira variável canônica (VC1) ou o equivalente a função discriminante linear de Fischer, identificado como eficiência bionutricional:

$\mathrm{VC} 1=-0,0584 * \mathrm{GPT}+1,2542 * \mathrm{CMS}$

O coeficiente do CMS é sempre positivo e o coeficiente para diferentes ganhos é sempre negativo, assim, animais com menor índice bionutricional são o mais eficientes (MARCONDES et al., 2011). Assim, como observado por Mello et al. (2009) quanto maior for o valor encontrado para a eficiência bionutricional, menor será a eficiência do animal, pois o coeficiente canônico associado ao ganho de peso foi negativo e o coeficiente associado ao consumo foi positivo.

No tocante à comparação entre médias de tratamentos para as variáveis $\mathrm{VC1}$, CA e EA verificaram-se as mesmas observações para as três variáveis, corroborando com o resultado, anteriormente obtido, para GPT e CMST (Tabela 1).

Ao analisar a distribuição de resíduos para VC1, CA e EA, pelo teste de Shapiro-Wilk, verificou-se ser razoável aceitar os resíduos como normalmente distribuídos. Porém ao analisar a probabilidade associada ao teste, verificou-se que enquanto que essa hipótese foi aceita para $8,14 \%$ na CA, a mesma foi aceita sob $41,37 \%$ para $\mathrm{VC} 1$, indicando assim que $\mathrm{VC1}$ possui distribuição mais assimétrica que $\mathrm{CA}$ e EA.

De fato, este resultado corrobora com as observações de Detmann et al. (2005) quando concluíram que a utilização da primeira variável canônica (índice bionutricional), garante a máxima discriminação entre os grupos experimentais. Além disso, segundo os autores o conceito de índice bionutricional mostra-se ser mais amplo do que as aplicadas à conversão alimentar ou à eficiência alimentar, visto que também incorpora os elementos relativos à intensidade da produção animal. Em adição, Marcondes et al. (2011) observaram que o índice bionutricional apresentou-se de forma diferente em relação à eficiência alimentar para os ganhos de peso vazio e carcaça de bovinos.

De acordo com Detmann et al. (2005), como o ganho de peso e o CMS são variáveis de distribuição normal, e a divisão entre elas gera uma variável de distribuição Cauchy. Assim, o mais adequado seria gerar uma variável de distribuição normal a partir da combinação linear de duas variáveis de distribuição normal, no caso ganho de peso e CMS.

Assim, de acordo com o índice, observouse que animais que começam o confinamento com mais de $350 \mathrm{~kg}$ são menos eficientes $(\mathrm{P}<0,05)$ que os animais com peso até $350 \mathrm{~kg}$ visto que apresentam maior CMS e menor GPT.

De fato, animais mais leves tem apresentado maior eficiência na conversão de alimento em carne (MYERS et al., 1999; SCHOONMAKER et al., 2002), em função dos menores requerimentos energéticos de mantença, $o$ que proporciona maior energia para ganho de peso (NRC, 2000).

Segundo Lanna \& Packer (1998), a deposição de proteína é menos eficiente energeticamente (Mcal/Mcal) que a de gordura, porém é mais eficiente em peso de tecido depositado ( $\mathrm{kg}$ músculo/Mcal ingerida), uma vez que, para cada unidade de ganho de proteína, aproximadamente três unidades de água são depositadas. Maldonado et al. (2007), também observaram uma menor eficiência para ganho de peso em animais mais pesados. 
Rev. Bras. Saúde Prod. Anim., Salvador, v.16, n.3, p.699-711 jul./set.., $2015 \quad \underline{\text { http://www.rbspa.ufba.br }}$ ISSN 15199940

As diferenças na composição química, calculada pelos ganhos em função do peso e da idade dos animais durante o confinamento, refletem sobre os custos de produção (GESUALDI JUNIOR et al., 2006). Costa et al. (2002) relataram que o aumento do peso de abate pode alterar o desempenho animal, alterando os custos do processo de terminação e consequentemente sua lucratividade.
Assim, no tocante à eficiência econômica (Tabela 2), observou-se que os grupos de variáveis de produção e variáveis financeiras se apresentam altamente correlacionados e somente os dois primeiros pares canônicos apresentaram relevância estatística $(\mathrm{P}<0,01)$.

Tabela 2. Coeficientes dos pares canônicos padronizados para os grupos de variáveis de produção e variáveis financeiras, com a correlação canônica $(\mathrm{R})$ e resultado do teste $x^{2}$

\begin{tabular}{lccc}
\hline \multirow{2}{*}{ Variáveis de produção } & \multicolumn{3}{c}{ Pares Canônicos } \\
\cline { 2 - 3 } & $\mathrm{V} 1$ & $\mathrm{~V} 2$ & $\mathrm{~V} 3$ \\
\hline Custo de produção por arroba (CP@) & $-0,0157$ & 0,1013 & $-0,1085$ \\
Valor de venda da arroba (VV@) & $-0,0349$ & $-0,6341$ & 0,9911 \\
Custo da diária (CD) & 0,0257 & 0,1330 & 0,6042 \\
Valor por cabeça na saída (VV/CAB) & 0,7269 & 0,9133 & $-0,4650$ \\
Lucro líquido do produto (LLíq) & 0,4671 & $-0,5664$ & $-0,3773$ \\
\hline Variáveis financeiras & & & 0,4274 \\
\hline Valor de compra por cabeça (VC/CAB) & 0,6390 & 0,7349 & $-0,5710$ \\
Lucro líquido por cabeça (LL/CAB) & 0,9085 & $-0,6665$ & 0,3363 \\
Valor por arroba de compra (VC@) & 0,4436 & $-1,2043$ & 0,15 \\
\hline $\mathrm{R}$ & 0,92 & 0,90 & $0,94 *$ \\
\hline $\mathrm{x}^{2}$ & $53,81 * *$ & $36,38 * *$ &
\end{tabular}

Avaliando-se a correlação das variáveis com o seu par canônico, observou-se que no primeiro par canônico (Tabela 3), nas variáveis de produção, com exceção do custo da diária (CD), todas as demais apresentaram alta correlação, porém a mesma variável foi a mais correlacionada do segundo par canônico.

No exame das variáveis financeiras, no primeiro par, o canônico VC@ foi a variável de menor relevância, porém a mesma mostrou-se altamente relevante no segundo par canônico, que por sua vez foi pouco correlacionado com $o$ LL/CAB. Assim, verificou-se que todas as variáveis apresentaram relevância com seus pares canônicos, com destaque para VV/CAB e LLíq e para $\mathrm{VC} / \mathrm{CAB}$.

A aquisição dos animais e a alimentação são os principais fatores que influenciam na rentabilidade dos confinamentos. De acordo com Ezequiel et al. (2006), o lucro do confinamento depende de diversos fatores, dentre os principais, estão o preço pago pelo boi magro e o peso inicial dos animais ao confinamento, e ainda, que o lucro com o ganho de peso do animal durante o período confinado pode representar em até $40 \%$ do lucro total desse sistema de terminação. 
Rev. Bras. Saúde Prod. Anim., Salvador, v.16, n.3, p.699-711 jul./set.., $2015 \quad \underline{\text { http://www.rbspa.ufba.br }}$ ISSN 15199940

Avaliando-se as correlações das variáveis de produção com as variáveis financeiras, verificou-se que todas as variáveis de produção afetaram o índice financeiro, com destaque para $\mathrm{VV} / \mathrm{CAB}$ e LLíq. Avaliando as variáveis de produção, também todas as variáveis financeiras afetaram $o$ índice de produção, com maior destaque para $\mathrm{VC} / \mathrm{CAB}$.

Tabela 3. Correlações das variáveis de produção e variáveis financeiras com o seu par canônico (dentro de grupo). Correlações das variáveis de produção com as variáveis canônicas das variáveis financeiras e entre as variáveis financeiras com as variáveis canônicas das variáveis de produção (Entre grupos)

\begin{tabular}{lcc}
\hline \multirow{2}{*}{ Variáveis de produção } & \multicolumn{2}{c}{ Pares Canônicos (dentro de grupos) } \\
\cline { 2 - 3 } Custo de produção por arroba (CP@) & V1 & V2 \\
Valor de venda da arroba (VV@) & $-0,6500$ & 0,3598 \\
Custo da diária (CD) & 0,5635 & $-0,3780$ \\
Valor por cabeça na saída (VV/CAB) & 0,1210 & 0,5849 \\
Lucro líquido do produto (LLíq) & 0,9067 & 0,3447 \\
\hline Variáveis financeiras & 0,7433 & $-0,5848$ \\
\hline Valor de compra por cabeça (VC/CAB) & & 0,4187 \\
Lucro líquido por cabeça (LL/CAB) & 0,6832 & $-0,0190$ \\
Valor por arroba de compra (VC@) & 0,5709 & $-0,5643$ \\
\hline & 0,1010 & 0,3227 \\
Variáveis de produção & Pares Canônicos (entre grupos) \\
\hline Custo de produção por arroba (CP@) & V1 \\
Valor de venda da arroba (VV@) & $-0,5987$ & $-0,3390$ \\
Custo da diária (CD) & 0,5190 & 0,5245 \\
Valor por cabeça na saída (VV/CAB) & 0,1115 & 0,3092 \\
Lucro líquido do produto (LLíq) & 0,8352 & $-0,5244$ \\
\hline Variáveis financeiras & 0,6846 & \\
\hline Valor de compra por cabeça (VC/CAB) & & 0,3755 \\
Lucro líquido por cabeça (LL/CAB) & 0,6293 \\
Valor por arroba de compra (VC@) & 0,5258 & $-0,0170$ \\
\hline
\end{tabular}

As variáveis zootécnicas e as variáveis financeiras são altamente correlacionadas (Tabela 4) e os dois pares canônicos foram estatisticamente relevantes $(\mathrm{P}<0,01)$. Do grupo de variáveis zootécnicas, tanto PCF quanto VC1 apresentaram importante contribuição para a primeira e segunda variável canônica, respectivamente.

A análise econômica da pecuária de corte é importante, pois o pecuarista passa a conhecer, com detalhes, os fatores de produção. A partir de estudos dessa natureza, identificam-se os pontos de estrangulamento, que permitem concentrar esforços gerenciais e tecnológicos para se obter sucesso na atividade e atingir a redução dos custos e a maximização de lucros (LOPES \& MAGALHÃES, 2005).

De acordo com Arboitte et al. (2004), períodos longos de confinamento, causados pelo baixo peso inicial dos animais, baixo ganho de peso, motivados por fatores genéticos e/ou baixa concentração energética da dieta, peso de abate elevado resultam em baixa eficiência econômica do processo. 
Rev. Bras. Saúde Prod. Anim., Salvador, v.16, n.3, p.699-711 jul./set.., $2015 \quad \underline{\text { http://www.rbspa.ufba.br }}$ ISSN 15199940

Tabela 4. Coeficientes dos pares canônicos padronizados para os grupos de variáveis de desempenho zootécnico e variáveis financeiras, com os seus pares canônicos, bem como a correlação canônica $(\mathrm{R})$ e resultado do teste $\mathrm{x}^{2}$

\begin{tabular}{lcc}
\hline \multirow{2}{*}{ Desempenho zootécnico } & \multicolumn{2}{c}{ Pares Canônicos } \\
\cline { 2 - 3 } & $\mathrm{V} 1$ & $\mathrm{~V} 2$ \\
\hline Variável canônica1 (VC1) & 0,2185 & 1,7576 \\
Peso corporal final (PCF) & 0,8120 & $-1,5741$ \\
\hline Variáveis financeiras & 0,9611 & 0,0363 \\
\hline Valor de compra por cabeça (VC/CAB) & 0,0890 & 1,1003 \\
Lucro líquido por cabeça (LL/CAB) & $-0,6474$ & 1,1256 \\
Valor por arroba de compra (VC@) & 0,99 & 0,60 \\
\hline $\mathrm{R}$ & $326,74 * *$ & $32,76^{* *}$ \\
\hline $\mathrm{x}^{2}$ &
\end{tabular}

Com relação ao grupo das variáveis financeiras, verifica-se importante contribuição das variáveis originais na formação da primeira e segunda variável canônica. A correlação das variáveis originais na formação de seus pares canônicos, por parte do grupo de variáveis zootécnicas (Tabela 5), tanto VC1 quanto PCF, mostraram altamente correlacionadas com seu par canônico, enquanto que para as varáveis financeiras, a maior correlação com o par canônico foi verificada para $\mathrm{VC} / \mathrm{CAB}$.

As variáveis do grupo zootécnico apresentaram expressiva correlação com a variável canônica do grupo de variáveis financeiras, enquanto que o $\mathrm{VC} / \mathrm{CAB}$ foi a que mais se correlacionou com a variável canônica do grupo zootécnico.

Tabela 5. Correlações das variáveis de desempenho zootécnico e variáveis financeiras, com o seus pares canônicos (Dentro de grupos). Correlações das variáveis de desempenho zootécnico com as variáveis canônicas das variáveis financeiras e entre variáveis financeiras com as variáveis canônicas do desempenho zootécnico (Entre grupos)

\begin{tabular}{lcc}
\hline \multirow{2}{*}{ Desempenho zootécnico } & \multicolumn{2}{c}{ Pares Canônicos (dentro de grupos) } \\
\cline { 2 - 3 } & $\mathrm{V} 1$ & $\mathrm{~V} 2$ \\
\hline Variável canônica1 & 0,8887 & 0,4585 \\
Peso corporal final (PCF) & 0,9924 & $-0,1234$ \\
\hline Variáveis financeiras & & 0,2797 \\
\hline Valor de compra por cabeça (VC/CAB) & 0,7424 & 0,4163 \\
Lucro líquido por cabeça (LL/CAB) & 0,3754 & 0,4724 \\
Valor por arroba de compra (VC@) & $-0,3909$ & 0,2755 \\
\hline Desempenho zootécnico & Pares Canônicos (entre grupos) \\
\hline Variável canônica1 & V1 \\
Peso corporal final (PCF) & 0,8810 & 0,0741 \\
\hline Variáveis financeiras & 0,9838 & 0,1681 \\
\hline Valor de compra por cabeça (VC/CAB) & & 0,2501 \\
Lucro líquido por cabeça (LL/CAB) & 0,7359 & 0,2839 \\
Valor por arroba de compra (VC@) & 0,3722 \\
\hline
\end{tabular}


Rev. Bras. Saúde Prod. Anim., Salvador, v.16, n.3, p.699-711 jul./set.., $2015 \quad \underline{\text { http://www.rbspa.ufba.br }}$ ISSN 15199940

Em estudo sobre análise econômica de diferentes estratégias de ganho de peso diário em bovinos confinados, Resende Filho (2003), ao simular 17 cenários, avaliou que o maior lucro econômico é obtido com a estratégia de ganho de PC médio diário de $1,05 \mathrm{Kg}$. Ou alternativamente, a estratégia de maior lucro foi caracterizada pelos 220 animais atingindo um peso ao abate médio de $480 \mathrm{Kg}$, em um período de 120 dias. $\mathrm{O}$ autor ainda ressalta que os custos com a compra do boi magro e a alimentação representam, respectivamente, $69,3 \%$ e $16,18 \%$ do custo total para essa estratégia de ganho de 1,05kg/dia. Tal fato mostra a importância em se otimizar a compra do boi magro e se minimizar os custos da alimentação dos animais para o sucesso do empreendimento.

Rodrigues Filho et al. (2002), destacaram como ordem de importância os seguintes componentes no custo total de produção: alimentação, preço do bezerro, mão-de-obra, outros (juros, reparos e depreciação) e os insumos veterinários. Neste mesmo trabalho, os autores mostram que a alimentação representa em torno de $48 \%$ e o valor do bezerro, aproximadamente $31 \%$ do custo total. Lopes \& Magalhães (2005) relataram que $68,4 \%$ dos custos operacionais foram representados pela aquisição de animais e a alimentação, $22,32 \%$.

Tanto VC1 quanto PCF, mostraram altamente correlacionadas com seu par canônico, enquanto em relação às variáveis financeiras, $\mathrm{VC} / \mathrm{CAB}$ apresentou maior correlação com a variável canônica (Tabela 5).

Ezequiel et al. (2006) apontam que a o período de aquisição dos animais e a melhor aquisição de insumos são de extrema importância, pois constituem o planejamento estratégico do confinamento, pois esses fatores equacionados e controlados contribuem para que o resultado seja satisfatório, de modo que o lucro proporcionado pelo ganho de peso durante o período de terminação seja maximizado pelo aumento na eficiência da utilização dos alimentos ou pelo menor preço de aquisição. Analisando os dois pares canônicos entre o grupo de variáveis zootécnicas com o grupo das variáveis de produção, verificou-se que ambos foram relevantes estatisticamente $(\mathrm{P}<0,01)$ e que ambos os grupos são correlacionados (Tabela 6).

Tabela 6. Coeficientes dos pares canônicos padronizados para os grupos de variáveis de desempenho zootécnico e variáveis de produção, com o seus pares canônico, bem como a correlação canônica $(\mathrm{R})$ e resultado do teste $\mathrm{x}^{2}$

\begin{tabular}{lcc}
\hline \multirow{2}{*}{ Desempenho zootécnico } & \multicolumn{2}{c}{ Pares Canônicos } \\
\cline { 2 - 3 } & V1 & V2 \\
\hline Variável canônica1 & 1,0007 & $-1,4614$ \\
Peso corporal final (PCF) & $-0,0009$ & 1,7712 \\
\hline Variáveis de produção & & $-0,0651$ \\
\hline Custo de produção por arroba (CP@) & 0,0409 & $-0,6636$ \\
Valor de venda da arroba (VV@) & $-0,3214$ & 0,5346 \\
Custo da diária (CD) & 0,0363 & 0,0863 \\
Valor por cabeça na saída (VV/CAB) & 1,1327 & $-0,3759$ \\
Lucro líquido do produto (LLíq) & 0,0279 & 0,62 \\
\hline $\mathrm{R}$ & 0,97 & $18,06^{* *}$
\end{tabular}


Rev. Bras. Saúde Prod. Anim., Salvador, v.16, n.3, p.699-711 jul./set.., $2015 \quad \underline{\text { http://www.rbspa.ufba.br }}$ ISSN 15199940

Avaliando de forma conjunta os dois pares canônicos (Tabela 7), verificou-se que, tanto $\mathrm{VC} 1$ quanto $\mathrm{PCF}$, foram altamente correlacionadas com os seus pares canônicos.

Das variáveis de produção, o CD foi a variável que menos contribui para a formação da variável canônica (Tabela 7). As duas variáveis zootécnicas afetaram de forma expressiva o grupo das variáveis de produção, enquanto que $\mathrm{VV} / \mathrm{CAB}$ foi a variável do grupo de produção que mais influenciou as variáveis zootécnicas.

O tempo de permanência dos animais confinados no Brasil tem variado de 90 e 120 dias. Os objetivos de se terminar bovinos em confinamento durante todo o ano são: garantir matéria-prima de qualidade para abastecer os frigoríficos, evitar que a estrutura, máquinas e funcionários do confinamento fiquem parados, o que resulta na diluição de custos.

Tabela 7. Correlações das variáveis de desempenho zootécnico e variáveis de produção, com os seus pares canônicos (Dentro de grupos). Correlações das variáveis de desempenho zootécnico com as variáveis canônicas das variáveis de produção e entre variáveis de produção com as variáveis canônicas do desempenho zootécnico (Entre grupos)

\begin{tabular}{lcc}
\hline \multirow{2}{*}{ Desempenho zootécnico } & \multicolumn{2}{c}{ Pares Canônicos (dentro de grupos) } \\
\cline { 2 - 3 } & $\mathrm{V} 1$ & $\mathrm{~V} 2$ \\
\hline Variável canônica1 & 1.0000 & 0.0005 \\
Peso corporal final (PCF) & 0.8251 & 0.5650 \\
\hline Variáveis de produção & & 0.5471 \\
\hline Custo de produção por arroba (CP@) & -0.3534 & -0.7051 \\
Valor de venda da arroba (VV@) & 0.3060 & 0.5877 \\
Custo da diária (CD) & 0.3920 & -0.2208 \\
Valor por cabeça na saída (VV/CAB) & 0.9625 & -0.7250 \\
Lucro líquido do produto (LLíq) & 0.2995 & V2 \\
\hline Desempenho zootécnico & Pares Canônicos (entre grupos) \\
\cline { 2 - 3 } & V1 & 0,0003 \\
Variável canônica1 & 0,9723 & 0,3519 \\
Peso corporal final (PCF) & 0,8023 & 0,3407 \\
\hline Variáveis de produção & & $-0,4391$ \\
\hline Custo de produção por arroba (CP@) & $-0,3436$ & 0,3660 \\
Valor de venda da arroba (VV@) & 0,2975 & $-0,1375$ \\
Custo da diária (CD) & 0,3811 & $-0,4515$ \\
Valor por cabeça na saída (VV/CAB) & 0,9359 & \\
Lucro líquido do produto (LLíq) & 0,2912 & \\
\hline
\end{tabular}

Alguns produtores confinam animais durante $\mathrm{o}$ ano todo, o que os torna muitas vezes, parceiros dos frigoríficos, porém é importante ressaltar que a arroba produzida no confinamento apresenta-se mais elevada em comparação a sistemas de terminação a pasto, o que exige redução de custos e eficiência produtiva para que não ocorram prejuízos.

A permanência do animal no confinamento varia de acordo com a qualidade do alimento e a maturidade do animal, pois na fase inicial da engorda, o animal apresenta maior deposição de musculatura e a exigência de energia é menor, quando comparada 
Rev. Bras. Saúde Prod. Anim., Salvador, v.16, n.3, p.699-711 jul./set.., $2015 \quad \underline{\text { http://www.rbspa.ufba.br }}$ ISSN 15199940

à fase final da terminação, segundo o NRC (2000) quando o animal necessita de maior aporte de energia para deposição de gordura.

Os dados analisados mostraram a correlação entre as variáveis zootécnicas e as variáveis econômicas dos animais terminados em confinamento e indicaram que a subtração dos custos sobre o valor da venda dos animais não mostraram com exatidão, a rentabilidade da atividade. Sobretudo porque o valor da arroba flutua ao longo do ano, não podendo ser considerado um parâmetro único para a análise econômica. Assim, a forma mais eficiente para se avaliar a rentabilidade da atividade é analisar a produtividade do animal durante o período de terminação, através do ganho de peso total. Além disso, diversos parâmetros devem ser analisados em conjunto, como o GMD, permanência de confinamento e eficiência alimentar.

É importante lembrar que tal prática não é padronizada em todo o país e engloba distintos sistemas de produção com parâmetros de rentabilidade variando de modo acentuado entre os confinadores. Sob essa ótica, é necessário adotar um planejamento da atividade tanto no que se refere à seleção dos animais a serem confinados (visando a maior homogeneidade quanto ao peso, idade e raça), quanto ao acompanhamento sistemático do desempenho dos animais no decorrer do processo, de modo a possibilitar a melhor estratégia quanto ao momento de comercialização na busca de maiores garantias em termos de resultados econômicos compensadores.

Independente da época do ano, animais com peso corporal até $350 \mathrm{~kg}$ ao início do confinamento apresentam menos consumo de matéria seca e maios ganho de peso e consequentemente maior eficiência bio-nutricional/econômica em relação à animais mais pesados no início do confinamento. $\mathrm{O}$ valor de compra por cabeça interfere altamente sobre valor por cabeça na saída que depende do consumo de matéria seca e do ganho de peso total para maximização da receita.

\section{REFERÊNCIAS}

ARBOITTE, M.Z.; RESTLE, J.;

ALVES FILHO, D.C.; BRONDANI, I.L.; SILVA, J.H.S.; NORNBERG, J.L.; KUSS, F. Desempenho em

confinamento de novilhos $5 / 8$ Nelore -

3/8 Charolês, abatidos em diferentes estádios de desenvolvimento. Revista

Brasileira de Zootecnia, v.33, n.4, p.947-958, 2004.

CHIZZOTTI, M.L.; CHIZZOTTI, F.H.M. Consumo e digestibilidade de plantas forrageiras In: REIS, R.A.; BERNERDES T.F.; SIQUEIRA, G.R. (Eds.). Forragicultura: Ciência, Tecnologia e Gestão dos Recursos Forrageiros. 1.ed. Jaboticabal, Gráfica Multipress, p.425-435,2014.

COSTA, E.C.; RESTLE, J.; PASCOAL, L.L.; VAZ, F.N.; ALVES FILHO, D.C.; ARBOITTE, M.Z. Desempenho de Novilhos Red Angus Superprecoces, Confinados e Abatidos com Diferentes Pesos. Revista Brasileira de

Zootecnia, v.31,n.1, 129-138, 2002.

CRUZ, G.M.; ESTEVES, S.N.; TULLIO, R.R.; ALENCAR, M.M.; OLIVEIRA, M.C.S. Peso de abate de machos não castrados para a produção de bovino jovem. 1 Desempenho em confinamento e custo de produção.

Revista Brasileira de Zootecnia, v.33, n.3, p.635-645, 2004. 
Rev. Bras. Saúde Prod. Anim., Salvador, v.16, n.3, p.699-711 jul./set.., $2015 \quad \underline{\text { http://www.rbspa.ufba.br }}$ ISSN 15199940

DETMANN, E.; CECON, P.R.; ANDREOTTI, M.O; RESENDE, F.D.; SOUSA, D.P.; PONCIANO, N.J.; CAMPOS, J.M.S.; SOUZA, P.M.; VITTORI, A. Avaliação da primeira variável canônica na avaliação de experimentos de desempenho produtivo com animais. Revista Brasileira de Zootecnia, v.34, n.6, p.2417-2426, 2005. Supl. 1.

EZEQUIEL, J.M.B.; GALATI, R.L.; MENDES, A.R.; FATURI, C.

Desempenho e características de carcaça de bovinos Nelore em confinamento alimentados com bagaço de cana-deaçúcar e diferentes fontes energéticas. Revista Brasileira de Zootecnia, v.35, n.5, p.2050-2057, 2006.

GESUALDI JÚNIOR, A.; QUEIROZ, A.C. de; RESENDE, F.D. de; ALLEONI, G.F.; RAZOOK, A.G.; FIGUEIREDO, L.A. de; GESUALDI, A.C.L. de S.; DETMANN, E. Desempenho produtivo e eficiência bioeconômica de bovinos Nelore e Caracu selecionados para peso aos 378 dias de idade recebendo alimentação à vontade ou restrita. Revista Brasileira de Zootecnia, v.35, p.576683, 2006.

\section{GUIDONI, A.L. Alternativas para} comparar tratamentos envolvendo o desempenho nutricional animal. Piracicaba, SP: ESALQ/USP, 1994. 105p. Tese (Doutorado) - Escola Superior de Agricultura "Luiz de Queiroz", 1994.

JOHNSON, R.A.; WICHERN, D.W. Applied multivariate statistical analysis. Prentice Hall, New jersey, $4^{\text {th }}$ ed. $1998.816 \mathrm{p}$.

KHATTREE R.; NAIK, D.N. Multivariate data reduction and discrimination with SAS software. Cary: SAS Institute, 2000. 558p.
LANNA, D.P.; PACKER, I.V.

Eficiência biológica e econômica de bovinos de corte. In: Workshop sobre qualidade da carne e melhoramento genético de bovinos, 1., 1998, São Carlos. Anais... São Carlos: EMBRAPA/FUNDEPEC, 1998. p.83104.

LOPES, M.A.; MAGALHÃES, G.P. Rentabilidade na terminação de bovinos de corte em confinamentos: um estudo de caso em 2003, na região oeste de Minas Gerais. Revista Ciência e Agrotecnologia, v.29, n.5, p.10391044, 2005.

MALDONADO, F.; QUEIROZ, A.C.; ALLEONI, G.F.; LEME, P.R.; BOIN, C.; RESENDE, F.D.; NARDON, R.F.; DEMARCHI, J.J.A.A.; OLIVEIRA, R.V. Desempenho e características da carcaça de bovinos de dois grupos genéticos, terminados em confinamento e abatidos com diferentes pesos.

Boletim da Indústria Animal, v.64, n.1, p.09-18., 2007.

MARCONDES, M.I.; VALADARES FILHO, S.C.; OLIVEIRA, I.M.; PAULINO, P.V.R.; VALADARES, R.F.D.; DETMANN, E. Eficiência alimentar de bovinos puros e mestiços recebendo alto ou baixo nível de concentrado. Revista Brasileira de Zootecnia, v.40, n.6, 1313-1324, 2011.

MELLO, R.; RESENDE, F.D.; QUEIROZ, A.C.; FARIA. M.H.; HENRIQUE, D.S.; MALDONADO, F. Bioeconomicity of the finishing phase on feedlot of crossbred young bulls slaughtered at different body weights. Revista Brasileira de Zootecnia, v.38, n.1,p.109-121, 2009 
Rev. Bras. Saúde Prod. Anim., Salvador, v.16, n.3, p.699-711 jul./set.., $2015 \quad \underline{\text { http://www.rbspa.ufba.br }}$ ISSN 15199940

MERTENS, D.R. Regulation of forage intake. In: FAHEY, G.C.; COLLINS, M.; MERTENS, D.R.; MOSER, L.E. (Eds). Forage quality evaluation and utilization. Madison: ASA, CSSA, SSSA, p. 450-493, 1994.

MERSMANN, H.J. Overview of the effects of beta-adrenergic receptor agonists on animal growth including mechanisms of action. Journal of Animal Science, v.76, n.1, p.160-172, 1998.

MYERS, S.E.; FAULKNER, D.B.; IRLAND, F.A.; BERGER, L.L.; PARRET, D.F. Production systems comparing early weaning to normal weaning with or without creep feeding for beef steers. Journal of Animal Science, v.77, p.300-310, 1999.

NATIONAL RESEARCH COUNCIL NRC. Nutrient requirements of beef cattle. $7^{\text {th }}$ ed. Washington: National Academy Press, 2000. 242p

PAZDIORA, R.D.; RESENDE, F.D.; FARIA, M.H.; SIQUEIRA, G.R.; ALMEIDA, G.B.S.; SAMPAIO, R.L.; PACHECO, P.S.; PRIETTO, M.S.R. Animal performance and carcass characteristics of Nellore young bulls fed coated or uncoated urea slaughtered at different weights. Revista Brasileira de Zootecnia, v.42, n.4, p.273-283, 2013.

REGAZZI, A.J. Análise multivariada: notas de aula INF 766. Viçosa, MG: Universidade Federal de Viçosa, 2000. v.2,

RESENDE FILHO, M.A. Avaliação econômica de diferentes estratégias de ganho de peso diário na terminação de bovinos em confinamento. In: CONGRESSO DA SOCIEDADE BRASILEIRA DE ECONOMIA,
ADMINISTRAÇÃO E SOCIOLOGIA RURAL, 46, 2008, Rio Branco, Acre. Anais... Rio Branco, Acre: SOBER, 2008.

RODRIGUES FILHO, M.; MANCIO, A.B.; GOMES, S.T.; SILVA. F.F.; LANA. R.P.; RODRIGUES, N.E.B; SOARES, C.A.; VELOSO, C.M. Avaliação Econômica do Confinamento de Novilhos de Origem Leiteira, Alimentados com Diferentes Níveis de Concentrado e de Cama de Frango.

Revista Brasileira de Zootecnia, v.31, n.5, p.2055-2069, 2002.

SCHOONMAKER, J.P.; LOERCH, S.C.; FLUHARTY, F.L.; ZERBY, H.N., TURNER, T.B. Effect of age at feedlot entry on performance carcass characteristics of bulls and steers. Journal of Animal Science, v.80, p.2247-2254, 2002.

SIMPFENDORFER, S. Relationship of body type and size, sex and energy intake to the body composition of cattle. 1974. 193p. Thesis (PhD) University of Cornell,. Ithaca.

VALADARES FILHO, S.C.; MARCONDES, M.I.; CHIZZOTTI, M.L.; PAULINO, P.V.R. Exigências nutricionais de zebuínos puros e cruzados: BRCorte. 2.ed. Viçosa, MG: Universidade Federal de Viçosa, 2010.

Data de recebimento: 01/09/2014

Data de aprovação: 15/09/2015 